\title{
Subacute combined degeneration of the spinal cord is associated with tripterygium glycoside tablet usage
}

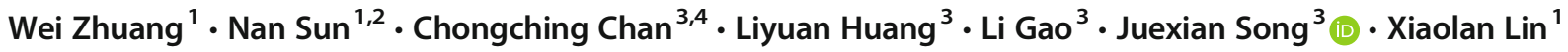

Received: 29 September 2018 / Accepted: 13 March 2019 / Published online: 21 March 2019

(C) Fondazione Società Italiana di Neurologia 2019

\begin{abstract}
Background Subacute combined degeneration (SCD) is a neurodegenerative disease caused by vitamin $\mathrm{B}_{12}$ deficiency. The lesions mainly involve the posterior cord, lateral cord, and peripheral nerves. Occasionally, the lesions also involve brain white matter and optic nerves in severe cases. Reports of drug-induced impaired absorption and metabolism of vitamin $\mathrm{B}_{12}$ resulting in SCD are scarce.

Introduction A patient developed SCD after long-term use of tripterygium glycoside tablets in the treatment of glomerulonephritis. However, after discontinuation and vitamin $\mathrm{B}_{12}$ treatment with tripterygium glycoside tablet, the symptoms of SCD were significantly resolved.

Conclusion Drug-induced SCD is a less commonly reported cause of the disease. Tripterygium glycoside tablets can induce adverse reactions in the digestive system, causing damage to absorption and metabolism of vitamin $\mathrm{B}_{12}$. Physicians should be aware of the possibility of tripterygium glycoside tablet-induced SCD after excluding more common causes such as inadequate dietary intake and impaired absorption due to gastrointestinal diseases or genetic disorders.
\end{abstract}

Keywords Subacute combined degeneration · Tripterygium glycoside tablets · Vitamin $\mathrm{B}_{12}$

\section{Introduction}

Tripterygium wilfordii is a traditional Chinese herb used for the treatment of "fire-wind-dampness" syndrome. Tripterygium glycoside tablets as a patent medicine were approved by the State Food and Drug Administration of the

Wei Zhuang and Nan Sun contributed equally to this work.

Juexian Song

juexiansong@163.com

$\triangle$ Xiaolan Lin

13522406044@163.com

1 Department of Pharmacy, Xuanwu Hospital of Capital Medical University, 45 Changchun St, Xicheng District, Beijing 100053, China

2 Pharmacy Department of Beijing Men Tou Gou Hospital, Beijing 102300, China

3 Department of Neurology, Xuanwu Hospital of Capital Medical University, 45 Changchun St, Xicheng District, Beijing 100053, China

4 Department of Medicine, Queen Elizabeth Hospital, Hong Kong, Hong Kong SAR, China
People's Republic of China, in which the main component is extracted from the rhizome of Tripterygium wilfordii [1]. The main bioactive components of tripterygium glycoside tablets are triptolide, tripterylide glycol, tripterone, and alkaloids, which have a variety of signal pathway activities. The antiinflammatory, immunosuppressive, anti-neoplastic, antioxidative, antimicrobial properties, and other effects of tripterygium glycoside tablets have been increasingly recognized. Clinical trials have shown that tripterygium glycoside tablets have a significant effect on the treatment of rheumatoid arthritis [2]. The tripterygium glycoside tablets have been used as the effective drug for malignancy such as Crohn's disease and HIV/ AIDS due to its various pharmacological activities [3, 4]. Moreover, tripterygium glycoside tablets have also been used in kidney disease because it can significantly reduce proteinuria and preserve renal function [5]. Tripterygium glycoside tablets are showing more therapeutic potential [5]. But it should be noted that tripterygium glycoside tablets have a high incidence of adverse reactions, including gastrointestinal discomfort, diarrhea, reproductive toxicity, hematological disorders, and liver damage [6]. Since tripterygium glycoside tablets have a narrow therapeutic window, its clinical application is often restricted by safety concerns [7]. Both the therapeutic 
and toxic effects of tripterygium glycoside tablets are mediated by the same mechanisms that induce apoptosis, oxidative stress, and the release of lactate dehydrogenase [8]. A metaanalysis conducted by Chi Zhang et al. showed that the main side effects of tripterygium glycoside tablets were gastrointestinal symptoms (13.3\%), adverse reproductive outcomes $(11.7 \%)$, skin reactions $(7.8 \%)$, and hematological and cardiovascular events $(6.5 \%)$ [9]. Some studies have shown that liver damage caused by tripterygium glycoside tablets has a dose-dependent relationship [10]. However, reports on digestive system adverse reactions are relatively rare and there are no reports on the effects of tripterygium glycoside tablets on gastrointestinal absorption and metabolism. In this article, we reported for the first time a patient who suffered from subacute combined degeneration of the spinal cord (SCD) because of vitamin $\mathrm{B}_{12}$ deficiency due to long-term use of tripterygium glycoside tablets.

\section{Case summary}

A 63-year-old man complained of subacute progressive bilateral hand numbness for more than 2 months and lower limb weakness for half a month. The patient's upper limb numbness gradually progressed in an incremental manner and was subsequently associated with a symptom of astereognosis. Half a month ago, the patient developed bilateral lower limb weakness and unsteadiness, which were more severe at night. The patient also complained about the recent memory impairment. He had a history of hypertension (well controlled with oral nifedipine and valsartan), type 2 diabetes mellitus (on longterm insulin aspart subcutaneous injection), and IgA nephropathy (treated with oral tripterygium glycoside tablets $10 \mu \mathrm{g}$ twice daily for about 10 years). He did not have chronic diarrhea and stomach surgery. The patient was not a vegetarian and had a balanced diet. The patient had a history of smoking and drinking for over 20 years, with smoking about 10 cigarettes and drinking about $100 \mathrm{ml}$ alcohol daily. But he had quit smoking and abstained from alcohol for more than 10 years. Cranial nerve examination results showed no abnormal sign. The patient had mild distal lower limb weakness (Medical Research Council grade 4+) with generalized hyporeflexia and bilateral positive Babinski sign. He had impairment of light touch, bilateral proprioception, pinprick sensation, and temperature sensation over bilateral lower limbs. He had no apparent cerebellar signs, and he walked with a stomping gait. He had a mild cognitive impairment.

Laboratory test results showed lower serum vitamin $\mathrm{B}_{12}$ level (103 pg/ml, normal range 180-914 pg/ml), albumin/ globulin ratio $(1.48 \%$, normal range $1.5-2.5 \%)$, and highdensity lipoprotein $(0.95 \mathrm{mmol} / \mathrm{l}$, normal range 1.08 $1.91 \mathrm{mmol} / \mathrm{l})$. In addition, indicators such as folic acid (20.88 ng/ml, normal range 3.1-19.9 $\mathrm{ng} / \mathrm{ml})$, mean corpuscular volume (108.3 fl, normal range 82.9-95 fl), mean corpuscular hemoglobin (35.3 pg, normal range 27-31 pg), carcinoembryonic antigen $(5.48 \mathrm{ng} / \mathrm{ml}$, normal range 0.01 $5.0 \mathrm{ng} / \mathrm{ml})$, neuron-specific enolase $(23.35 \mathrm{ng} / \mathrm{ml}$, normal range $0.01-17.0 \mathrm{ng} / \mathrm{ml})$, glycosylated hemoglobin $(6.2 \%$, normal range $4.0-6.0 \%)$, and the number of neutrophils $(8.81 \times$ $10^{9} / 1$, normal range $1.8-6.4 \times 10^{9} / 1$ ) were abnormally elevated. The other indicators were within the normal range. Regular etiologies of vitamin $\mathrm{B}_{12}$ deficiency such as anemia, malnutrition, malabsorption, and other causes of SCD such as $\mathrm{N}_{2} \mathrm{O}$ were excluded due to patient history and laboratory tests. Differential diagnosis excluded diseases such as demyelinating, infectious, neoplastic, autoimmune, and nutritional diseases. The patient had no discomfort in the gastrointestinal tract, so there was no gastrointestinal endoscopy and pathological examination. Moreover, magnetic resonance imaging (MRI) of the cervical cord showed hyperintensity on the dorsal column and intervertebral disc herniation of cervical $\mathrm{C} 2$ C6 levels (Fig. 1a, b). Considering the patient's clinical impairment of pyramidal tract, dorsal column, and peripheral nerves, as well as his neuroimaging results and low serum vitamin $\mathrm{B}_{12}$ level, he was diagnosed as $\mathrm{SCD}$.

The patient's bilateral lower limb motor function defects were improved 1 month after the cessation of tripterygium glycoside tablet administration and treatment with vitamin $\mathrm{B}_{12}$. The vitamin $\mathrm{B}_{12}$ treatment lasted for 8 weeks with a daily injection dosage of $1000 \mu \mathrm{g}$. The vitamin $\mathrm{B}_{12}$ levels were gradually increased with improvement of the patient clinical symptoms. Eight weeks after the discontinuation of tripterygium glycoside tablets, the measurement of laboratory

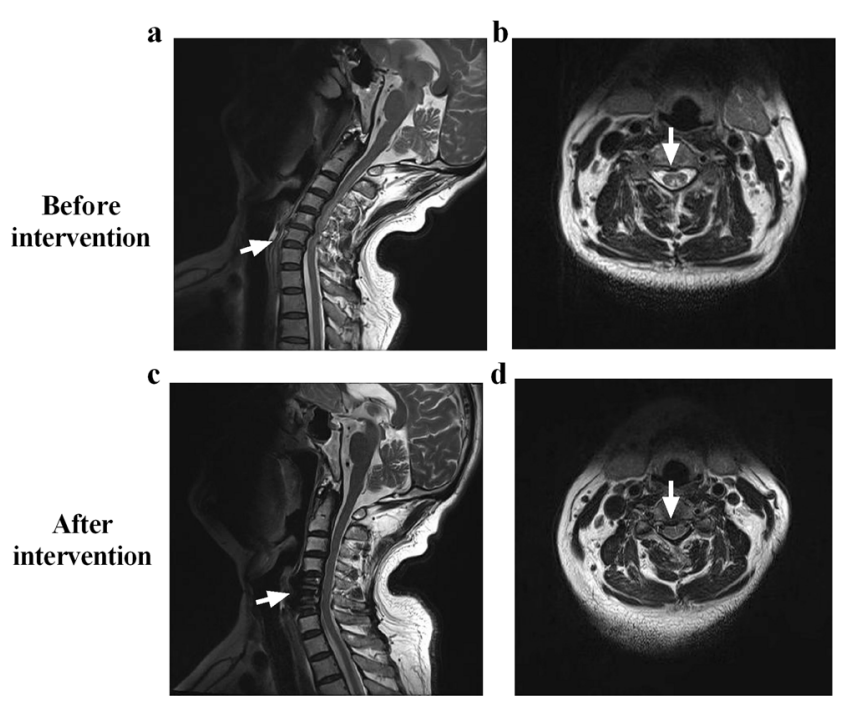

Fig. 1 MRI of a patient presenting with subacute combined degeneration before and after intervention. The patient presenting with SCD depicts hyperintensity involving the posterior part of the cervical spinal cord on sagittal (a) image and inverted "V" sign on axial section (b, arrow). After intervention, there was disappearance of the hyperintensity involving the posterior part of the cervical spinal cord on sagittal (c) image and inverted "V" sign on axial section (d, arrow) 
indicators and MRI was performed. The results showed that the patient had a normal range of serum vitamin B12 levels $(680 \mathrm{pg} / \mathrm{ml})$, mean red blood cell volume $(88.9 \mathrm{fl})$, and mean red blood cell hemoglobin (29.3 pg). Furthermore, the MRI results showed that the horizontal signal of cervical C2-C6 was normalized (Fig. 1c, d), suggesting that the lesion disappeared after the discontinuation of tripterygium glycoside tablets followed by supplementation of vitamin $\mathrm{B}_{12}$. Thus, we inferred that SCD is associated with tripterygium glycoside tablets usage. The patient was discharged after 6 weeks and after 6 months follow-up, no recurrence was observed.

\section{Discussion}

$\mathrm{SCD}$ is a neurodegenerative disease induced by vitamin $\mathrm{B}_{12}$ deficiency. The lesions mainly involve the posterior cord, lateral cord, and peripheral nerves, but in some serious cases, the lesions will also affect the brain white matter and optic nerve [11]. Vitamin $B_{12}$ in the human body can only be ingested through animal food, and there are three kinds of vitamin $\mathrm{B}_{12}$ cofactors in the body, which can be used as coenzyme for the metabolism of folic acid (one carbon unit) and the tricarboxylic acid cycle [12]. In 1981, Scott et al. found that supplementation with methionine could effectively prevent the development of SCD in monkeys exposed to $\mathrm{N}_{2} \mathrm{O}$, which provided proof that the neurological impairment caused by vitamin $\mathrm{B}_{12}$ deficiency were related to impaired methylation [13]. It is well-known that any factor affecting the synthesis and activity of methyl vitamin $\mathrm{B}_{12}$ and adenosine vitamin $\mathrm{B}_{12}$ may result in SCD.

The main causes of vitamin $\mathrm{B}_{12}$ deficiency are genetic disorders affecting the metabolism of vitamin $\mathrm{B}_{12}$, insufficient intake of vitamin $B_{12}$, which is mostly seen in vegetarian diet, malabsorption caused by gastrointestinal disorders, as well as improper use of drugs. The latter three causes-induced vitamin $\mathrm{B}_{12}$ deficiencies can be quickly and effectively treated by vitamin $\mathrm{B}_{12}$ supplementation. Normally, the body stores up about 2 to $5 \mu \mathrm{g}$ of vitamin $\mathrm{B}_{12}$ and about 0.1 to $0.2 \%$ of stored vitamin $B_{12}$ is used daily. Therefore, it usually takes 3 to 6 years for serious defects to occur [14].

Our patient was not a vegetarian and had a balanced diet, with no history of gastrointestinal or related genetic disorders, but he still developed vitamin $\mathrm{B}_{12}$ deficiency, which led to SCD. He did not frequently take metformin, and further analysis of his drug history showed that tripterygium glycoside tablets were the cause of vitamin $\mathrm{B}_{12}$ deficiency. It is likely that long-term use of tripterygium glycoside tablets may lead to vitamin $B_{12}$ malabsorption, resulting in vitamin $B_{12}$ deficiency and complications caused by vitamin B12 deficiency.

Tripterygium glycoside tablets are a fat-soluble mixture extracted from the rhizome of Tripterygium wilfordii, and its pharmacological activity is produced by various components, including diterpenoids, triterpenoids, and sesquiterpene alkaloids [15]. It has been found that tripterygium glycoside tablets have anti-inflammatory, immunosuppressive or immunoregulation, anti-neoplastic, and antimicrobial effects. Tripterygium glycoside tablets have been proven to have therapeutic value in the treatment of rheumatoid arthritis, glomerulonephritis, systemic lupus erythematosus, and other autoimmune diseases. Studies have shown that tripterygium glycoside tablets have significant therapeutic effects on nephrotic syndrome, IgA nephropathy, and chronic glomerulonephritis, but it is often accompanied by frequent adverse reactions [5]. Tripterygium glycoside tablets exert significant toxic effects on the reproductive system of male SD rats, and the toxicity is time- and dose-dependent [16, 17]. Furthermore, tripterygium glycoside tablets lead to acute hepatic injury, myocardial damage, and gastrointestinal inflammatory changes [18, 19]. Tripterygium glycoside tablets and its preparations also have obvious gastrointestinal toxicity, mainly manifested as nausea, diarrhea, and liver impairment [9]. Clinical studies have confirmed that tripterygium glycoside tablets can affect a variety of metabolic pathways, including lipid metabolism, tricarboxylic acid cycle, digestion, and absorption [20-22]. High dose of tripterygium glycoside tablets can cause a timedependent toxicity, such as disorder of the metabolic regulation network, enhancement of amino acid and choline metabolism, and alteration of the intestinal flora [23]. Based on the many side effects of tripterygium glycoside tablets, especially gastrointestinal inflammatory and toxicity, thus, long-term use of tripterygium glycoside tablets may cause damage to normal gastrointestinal mucosal, resulting in malabsorption. Moreover, the toxicity of tripterygium glycoside tablets is different between normal and hyperimmune bodies [20]. These side effects of tripterygium glycoside tablets on gastrointestinal structure and intestinal flora may be one of the possible mechanisms leading to malabsorption and thus vitamin $\mathrm{B}_{12}$ deficiency. Metabolic profiling indicated that a significant increase in the content of urinary amino acids such as leucine, lysine, acetyl-lycine, tyrosine, and glutamine after long-term high-dose use of tripterygium glycoside tablets suggests that tripterygium glycoside tablets can cause abnormal amino acids metabolism [24]. Furthermore, it is also a possible factor that may lead to SCD [22]. Because vitamin $B_{12}$ is the key coenzyme in the methionine metabolic pathway, it is closely related to the metabolic pathway of amino acids. The enhancement of amino acid metabolism may lead to a large amount of coenzyme depletion, increased consumption of vitamin $\mathrm{B}_{12}$, decreased serum vitamin $\mathrm{B}_{12}$ level, and reduced storage of vitamin $\mathrm{B}_{12}$ in the body, leading to the symptoms of the SCD. 
This work presents some limitations. The cessation of the trypterygium tablets and the disappearance of the symptoms suggested a link, but considering the re-supplementation of vita$\min B_{12}$, it could be the sole reason for the improvement of SCD.

\section{Conclusion}

We reported a patient suffering from vitamin $\mathrm{B}_{12}$ deficiency, which was associated with the long-term use of tripterygium glycoside tablets. This case report emphasizes that the symptoms of gastrointestinal toxicity caused by tripterygium glycoside tablets may not only manifest as nausea, vomiting, and liver impairment. Further research is needed to determine a definite causal relationship between tripterygium glycoside tablets and vitamin $\mathrm{B}_{12}$ deficiency. It is also necessary to further confirm the exact mechanism of vitamin $\mathrm{B}_{12}$ deficiency caused by tripterygium glycoside tablets in the future.

Funding This work was supported by the Foundation for Beijing Science \& Technology Development of TCM (JJ2016-10) and Beijing Municipal commission of Health and Family Planning (No. PXM2017 026283 000002).

\section{Compliance with ethical standards}

Conflict of interest The authors declare that they have no conflict of interest.

\section{References}

1. Zhang Y, Jiang Z, Xue M, Zhang S, Wang Y, Zhang L (2012) Toxicogenomic analysis of the gene expression changes in rat liver after a 28-day oral Tripterygium wilfordii multiglycoside exposure. J Ethnopharmacol 141(1):170-177

2. Pyatt DW, Yang Y, Mehos B, le A, Stillman W, Irons RD (2000) Hematotoxicity of the chinese herbal medicine Tripterygium wilfordii Hook F in CD34-positive human bone marrow cells. Mol Pharmacol 57(3):512-518

3. Kannaiyan R, Shanmugam MK, Sethi G (2011) Molecular targets of celastrol derived from Thunder of God Vine: potential role in the treatment of inflammatory disorders and cancer. Cancer Lett 303(1):9-20

4. Zhu W, Li Y, Gong J, Zuo L, Zhang W, Cao L, Gu L, Guo Z, Li N, Li J (2015) Tripterygium wilfordii Hook. F. versus azathioprine for prevention of postoperative recurrence in patients with Crohn's disease: a randomized clinical trial. Dig Liver Dis 47(1):14-19

5. Wang D, Zhao XH, Cui Y, Zhang TT, Wang F, Hu YH (2018) Efficacy and safety of Tripterygium wilfordii Hook F for CKD in Mainland China: A systematic review and meta-analysis. Phytother Res 32(3):436-451
6. Hong Y, Gui Z, Cai X, Lan L (2016) Clinical efficacy and safety of tripterygium glycosides in treatment of stage IV diabetic nephropathy: A meta-analysis. Open Med (Wars)11(1): 611-617

7. Li XJ, Jiang ZZ, Zhang LY (2014) Triptolide: progress on research in pharmacodynamics and toxicology. J Ethnopharmacol 155(1):67-79

8. Fan D, Parhira S, Zhu GY, Jiang ZH, Bai LP (2016) Triterpenoids from the stems of Tripterygium regelii. Fitoterapia 113:69-73

9. Zhang $\mathrm{C}$ et al (2016) Safety profiles of Tripterygium wilfordii Hook F: a systematic review and meta-analysis. Front Pharmacol 7:402

10. Li HG, Ji W, Su JM (2012) Literature research of the hepatotoxicity of glucoside tripterygium total and its synergism and toxicity reducing effects. Zhongguo Zhong Xi Yi Jie He Za Zhi 32(3):415-418

11. Hemmer B, Glocker FX, Schumacher M, Deuschl G, Lucking CH (1998) Subacute combined degeneration: clinical, electrophysiological, and magnetic resonance imaging findings. J Neurol Neurosurg Psychiatry 65(6):822-827

12. Smith AD, Warren MJ, Refsum H (2018) Vitamin B12, in Advances in food and nutrition research. Elsevier, Amsterdam, pp 215-279

13. Scott $J$ et al (1981) Pathogenesis of subacute combined degeneration: a result of methyl group deficiency. Lancet 318(8242):334-337

14. Chatthanawaree W (2011) Biomarkers of cobalamin (vitamin B12) deficiency and its application. J Nutr Health Aging 15(3):227-231

15. $\mathrm{Qu} \mathrm{L}$, et al (2015) Comprehensive two-dimensional liquid chromatography coupled with quadrupole time-of-flight mass spectrometry for chemical constituents analysis of tripterygium glycosides tablets. J Chromatogr A 1400:65-73

16. Leng Q, Cui RQ, Lu B (2011) Effects of multi-glycosides of tripterygium wilfordii on histological structures and c-kit expression in testes of pubertal rats. Zhongguo Dang Dai Er Ke Za Zhi 13(10):832-836

17. Jing X, et al (2017) Toxic effects of Tripterygium wilfordii Hook F on the reproductive system of adolescent male rats. Biomed Pharmacother 95:1338-1345

18. Peng B, Miao MS, Wang YL (2003) Initial discussion of mice acute hepatic injury caused by Tripterygium glycosides]. Zhongguo Zhong Yao Za Zhi 28(11):1067-1070

19. Huang GZ, Li L, Liu L (2009) Pathological study on autopsy died of Tripterygium intoxication-report of 4 cases. Zhongguo Zhong Xi Yi Jie He Za Zhi 29(2):165-168

20. Li J, Lu Y, Xiao C, Lu C, Niu X, He X, Zhao H, Tan Y, Lu A (2011) Comparison of toxic reaction of Tripterygium wilfordii multiglycoside in normal and adjuvant arthritic rats. J Ethnopharmacol 135(2):270-277

21. Ma ZJ, et al (2015) Tripterygium Glycosides Tablet Ameliorates Renal Tubulointerstitial Fibrosis via the Toll-Like Receptor 4/ Nuclear Factor Kappa B Signaling Pathway in High-Fat Diet Fed and Streptozotocin-Induced Diabetic Rats. J Diabetes Res 2015: 390428

22. Su M, et al (2011) Study on influence of glucoside Tripterygium total tablets on metabolism in rats by NMR metabonomic technique. Zhongguo Zhong Yao Za Zhi 36(11):1449-1453

23. Chen M, Ni Y, Duan H, Qiu Y, Guo C, Jiao Y, Shi H, Su M, Jia W (2008) Mass spectrometry-based metabolic profiling of rat urine associated with general toxicity induced by the multiglycoside of Tripterygium wilfordii Hook. F. Chem Res Toxicol 21(2):288-294

24. Minjun C et al (2008) Mass spectrometry-based metabolic profiling of rat urine associated with general toxicity induced by the multiglycoside of Tripterygium wilfordii Hook. F. Chem Res Toxicol 21(2):288

Publisher's note Springer Nature remains neutral with regard to jurisdictional claims in published maps and institutional affiliations. 